\title{
Several identities containing central binomial coefficients and derived from series expansions of powers of the arcsine function
}

\author{
Feng $Q^{a, b, c}$, Chao-Ping Chen ${ }^{d}$, Dongkyu Lim ${ }^{e, f}$ \\ ${ }^{a}$ College of Mathematics and Physics, Inner Mongolia University for Nationalities, Tongliao 028043, Inner Mongolia, China \\ bInstitute of Mathematics, Henan Polytechnic University, Jiaozuo 454010, Henan, China \\ 'School of Mathematical Sciences, Tianjin Polytechnic University, Tianjin 300387, China \\ ${ }^{d}$ School of Mathematics and Informatics, Henan Polytechnic University, Jiaozuo 454010, Henan, China \\ e Department of Mathematics Education, Andong National University, Andong 36729, Republic of Korea \\ ${ }^{f}$ Corresponding author
}

\begin{abstract}
In the paper, with the aid of the series expansions of the square or cubic of the arcsine function, the authors establish several possibly new combinatorial identities containing the ratio of two central binomial coefficients which are related to the Catalan numbers in combinatorial number theory.

Keywords: identity; product; ratio; central binomial coefficient; power series expansion; arcsine function; square; cubic; generating function; Catalan number

2010 MSC: Primary 05A10, 11B65; Secondary 05A15, 11B83, 26A09, 41A58
\end{abstract}

\section{Introduction}

The sequence of central binomial coefficients $\left(\begin{array}{c}2 n \\ n\end{array}\right)$ for $n \geq 0$ is classical, simple, and elementary. This sequence has attracted many mathematicians who have published a number of papers such as [3, 7, 10, 11, 17, 20, 27, 44, 46] and closely related references therein. It is worth to mentioning that, the integral representation

$$
\left(\begin{array}{c}
2 n \\
n
\end{array}\right)=\frac{1}{\pi} \int_{0}^{\infty} \frac{1}{\left(1 / 4+s^{2}\right)^{n+1}} \mathrm{~d} s
$$

Email addresses: qifeng618@gmail.com, qifeng618@hotmail.com, qifeng618@qq.com(Feng Qi), chenchaoping@sohu.com (Chao-Ping Chen), dgrim84@gmail.com, dklim@andong.ac.kr (Dongkyu Lim) 
was derived in [35, Section 4.2].

In this paper, with the help of the power series expansion

$$
\arcsin x=\sum_{\ell=0}^{\infty} \frac{1}{2^{2 \ell}}\left(\begin{array}{c}
2 \ell \\
\ell
\end{array}\right) \frac{x^{2 \ell+1}}{2 \ell+1}, \quad|x|<1,
$$

see [1, 4.4.40] and [2, p. 121, 6.41.1], the series expansion

$$
(\arcsin x)^{2}=\frac{1}{2} \sum_{\ell=1}^{\infty} \frac{(2 x)^{2 \ell}}{\ell^{2}\left(\begin{array}{c}
2 \ell \\
\ell
\end{array}\right)}, \quad|x|<1,
$$

which or its variants can be found in [2, p. 122, 6.42.1], [4, pp. 262-263, Proposition 15], [5, pp. 50-51 and p. 287], [6, p. 384], [12, Lemma 2], [15, p. 308], [16, pp. 88-90], [18, p. 61, 1.645], [24, p. 453], [33, Section 6.3], [48, p. 59, (2.56)], or [50, p. $676,(2.2)]$, and the power series expansion

$$
(\arcsin x)^{3}=3 ! \sum_{\ell=0}^{\infty}[(2 \ell+1) ! !]^{2}\left[\sum_{k=0}^{\ell} \frac{1}{(2 k+1)^{2}}\right] \frac{x^{2 \ell+3}}{(2 \ell+3) !}, \quad|x|<1,
$$

which or its variants can be found in [2, p. 122, 6.42.2], [4, pp. 262-263, Proposition 15], [9, p. 188, Example 1], [15, p. 308], [16, pp. 88-90], or [18, p. 61, 1.645], we will establish several identities involving the product $\left(\begin{array}{c}2 k \\ k\end{array}\right)\left(\begin{array}{c}2(n-k) \\ n-k\end{array}\right)$ or the ratio $\frac{\left(\begin{array}{c}2 k \\ k\end{array}\right)}{\left(\begin{array}{c}2(n-k+1) \\ n-k+1\end{array}\right)}$ of two central binomial coefficients $\left(\begin{array}{c}2 k \\ k\end{array}\right)$ and $\left(\begin{array}{c}2(n-k) \\ n-k\end{array}\right)$ for $0 \leq k \leq n$.

\section{Alternative proofs of a known combinatorial identity}

In this section, by means of the series expansions $(1.1)$ and $(1.2)$, we give two alternative proofs of a known combinatorial identity. This means that the method used in this paper is better.

Theorem 2.1 ([45, p. 77, (3.96)]). For $n \geq 0$, we have

$$
\sum_{k=0}^{n} \frac{1}{2 k+1}\left(\begin{array}{c}
2 k \\
k
\end{array}\right)\left(\begin{array}{c}
2(n-k) \\
n-k
\end{array}\right)=\frac{2^{4 n}}{(2 n+1)\left(\begin{array}{c}
2 n \\
n
\end{array}\right)} .
$$

First proof. From (1.1), it follows that

$$
\frac{1}{2} \arcsin (2 x)=\sum_{k=0}^{\infty} \frac{1}{2 k+1}\left(\begin{array}{c}
2 k \\
k
\end{array}\right) x^{2 k+1}, \quad|x|<\frac{1}{2}
$$

and, by differentiation,

$$
\frac{1}{\sqrt{1-4 x^{2}}}=\sum_{k=0}^{\infty}\left(\begin{array}{c}
2 k \\
k
\end{array}\right) x^{2 k}, \quad|x|<\frac{1}{2}
$$

Therefore, we obtain

$$
\frac{\arcsin (2 x)}{2 x \sqrt{1-4 x^{2}}}=\left[\sum_{k=0}^{\infty} \frac{1}{2 k+1}\left(\begin{array}{c}
2 k \\
k
\end{array}\right) x^{2 k}\right] \sum_{k=0}^{\infty}\left(\begin{array}{c}
2 k \\
k
\end{array}\right) x^{2 k}=\sum_{n=0}^{\infty}\left[\sum_{k=0}^{n} \frac{1}{2 k+1}\left(\begin{array}{c}
2 k \\
k
\end{array}\right)\left(\begin{array}{c}
2(n-k) \\
n-k
\end{array}\right)\right] x^{2 n} .
$$

On the other hand, by virtue of the series expansion 1.2 , we acquire

$$
\begin{gathered}
\frac{\arcsin (2 x)}{2 x \sqrt{1-4 x^{2}}}=\frac{1}{8 x} \frac{\mathrm{d}}{\mathrm{d} x}\left([\arcsin (2 x)]^{2}\right)=\frac{1}{8 x} \frac{\mathrm{d}}{\mathrm{d} x} \sum_{n=0}^{\infty} \frac{2^{2 n+1}(n !)^{2}}{(2 n+2) !}(2 x)^{2 n+2} \\
=\frac{1}{8 x} \sum_{n=0}^{\infty} \frac{2^{2 n+2}(n !)^{2}}{(2 n+1) !}(2 x)^{2 n+1}=\sum_{n=0}^{\infty} \frac{2^{4 n}(n !)^{2}}{(2 n+1) !} x^{2 n} .
\end{gathered}
$$


Comparing 2.2 with 2.3 and equating coefficients of $x^{2 n}$, we obtain

$$
\sum_{k=0}^{n} \frac{1}{2 k+1}\left(\begin{array}{c}
2 k \\
k
\end{array}\right)\left(\begin{array}{c}
2(n-k) \\
n-k
\end{array}\right)=\frac{2^{4 n}(n !)^{2}}{(2 n+1) !}=\frac{2^{4 n}}{(2 n+1)\left(\begin{array}{c}
2 n \\
n
\end{array}\right)} .
$$

The identity 2.1 is thus proved. The first proof of Theorem 2.1 is complete.

Second proof. Differentiating on both sides of 1.2 and rearranging give

$$
\frac{2 x \arcsin x}{\sqrt{1-x^{2}}}=\sum_{\ell=1}^{\infty} \frac{(2 x)^{2 \ell}}{\ell\left(\begin{array}{c}
2 \ell \\
\ell
\end{array}\right)}, \quad|x|<1,
$$

which or its variants can also be found in [2, p. 122, 6.42.5], [6, p. 384], [8, p. 161], [24, p. 452, Theorem], and [33, Section 6.3, Theorem 21, Sections 8 and 9]. Replacing $x$ by $2 x$ in (2.4) and rearranging yield

$$
\begin{gathered}
\frac{\arcsin (2 x)}{2 x} \frac{1}{\sqrt{1-4 x^{2}}}=\frac{1}{8 x^{2}} \frac{4 x \arcsin (2 x)}{\sqrt{1-4 x^{2}}}=\frac{1}{8 x^{2}} \sum_{\ell=1}^{\infty} \frac{(4 x)^{2 \ell}}{\ell\left(\begin{array}{c}
2 \ell \\
\ell
\end{array}\right)} \\
=\frac{1}{8 x^{2}} \sum_{n=0}^{\infty} \frac{(4 x)^{2(n+1)}}{(n+1)\left(\begin{array}{c}
2(n+1) \\
n+1
\end{array}\right)}=\sum_{n=0}^{\infty} \frac{2^{4 n+1}}{(n+1)\left(\begin{array}{c}
2(n+1) \\
n+1
\end{array}\right)} x^{2 n}
\end{gathered}
$$

for $|x|<\frac{1}{2}$. Comparing 2.2 with 2.5 and equating coefficients of $x^{2 n}$, we obtain

$$
\sum_{k=0}^{n} \frac{1}{2 k+1}\left(\begin{array}{c}
2 k \\
k
\end{array}\right)\left(\begin{array}{c}
2(n-k) \\
n-k
\end{array}\right)=\frac{2^{4 n+1}}{(n+1)\left(\begin{array}{c}
(n+1) \\
n+1
\end{array}\right)}=\frac{2^{4 n}}{(2 n+1)\left(\begin{array}{c}
2 n \\
n
\end{array}\right)} .
$$

The identity 2.1 is proved again. The second proof of Theorem 2.1 is complete.

\section{Three possibly new combinatorial identities}

In this section, by virtue of those three series expansions $(1.1),(1.2)$, and $(1.3)$, we establish three possibly new combinatorial identities involving the ratio $\frac{\left(\begin{array}{c}2 k \\ k\end{array}\right)}{\left(\begin{array}{c}2(n-k+1) \\ n-k+1\end{array}\right)}$ in terms of the trigamma function $\psi^{\prime}\left(n+\frac{3}{2}\right)$, where $\psi(x)$ is the digamma function defined by the logarithmic derivative $\psi(x)=[\ln \Gamma(x)]^{\prime}=\frac{\Gamma^{\prime}(x)}{\Gamma(x)}$ of the classical Euler gamma function

$$
\Gamma(z)=\int_{0}^{\infty} t^{z-1} e^{-t} \mathrm{~d} t, \quad \Re(z)>0 .
$$

This means that the method used in this paper is extensively applicable. For more information on the gamma function $\Gamma(x)$ and polygamma functions $\psi^{(k)}(x)$ for $k \geq 0$, please refer to [1, pp. 255-293, Chapter 6] or the papers [32, 36] and closely related references therein.

Theorem 3.1. For $n \geq 0$, we have

$$
\begin{aligned}
\sum_{k=0}^{n} \frac{1}{2^{4 k}(2 k+1)(n-k+1)^{2}} \frac{\left(\begin{array}{c}
2 k \\
k
\end{array}\right)}{\left(\begin{array}{c}
2(n-k+1) \\
n-k+1
\end{array}\right)} & =\frac{3[(2 n+1) ! !]^{2}}{2^{2 n+3}(2 n+3) !}\left[\pi^{2}-2 \psi^{\prime}\left(n+\frac{3}{2}\right)\right] \\
\sum_{k=0}^{n} \frac{1}{2^{4 k}(n-k+1)^{2}} \frac{\left(\begin{array}{c}
2 k \\
k
\end{array}\right)}{\left(\begin{array}{c}
2(n-k+1) \\
n-k+1
\end{array}\right)} & =\frac{[(2 n+1) ! !]^{2}}{2^{2 n+3}(2 n+2) !}\left[\pi^{2}-2 \psi^{\prime}\left(n+\frac{3}{2}\right)\right]
\end{aligned}
$$

and

$$
\sum_{k=0}^{n} \frac{1}{2^{4 k}(2 k+1)(n-k+1)} \frac{\left(\begin{array}{c}
2 k \\
k
\end{array}\right)}{\left(\begin{array}{c}
2(n-k+1) \\
n-k+1
\end{array}\right)}=\frac{[(2 n+1) ! !]^{2}}{2^{2 n+3}(2 n+2) !}\left[\pi^{2}-2 \psi^{\prime}\left(n+\frac{3}{2}\right)\right] .
$$


Proof. Differentiating on both sides of 1.3 gives

$$
\frac{(\arcsin x)^{2}}{\sqrt{1-x^{2}}}=2 ! \sum_{n=0}^{\infty}[(2 n+1) ! !]^{2}\left[\sum_{k=0}^{n} \frac{1}{(2 k+1)^{2}}\right] \frac{x^{2 n+2}}{(2 n+2) !}, \quad|x|<1 .
$$

On the other hand, we have

$$
\begin{aligned}
& (\arcsin x)^{3}=(\arcsin x)(\arcsin x)^{2} \\
& =\left[\sum_{k=0}^{\infty} \frac{1}{2^{2 k}}\left(\begin{array}{c}
2 k \\
k
\end{array}\right) \frac{x^{2 k+1}}{2 k+1}\right]\left[\frac{1}{2} \sum_{m=1}^{\infty} \frac{(2 x)^{2 m}}{m^{2}\left(\begin{array}{c}
2 m \\
m
\end{array}\right)}\right] \\
& =\frac{x^{3}}{2}\left[\sum_{k=0}^{\infty} \frac{1}{(2 k+1) 2^{2 k}}\left(\begin{array}{c}
2 k \\
k
\end{array}\right) x^{2 k}\right]\left[\sum_{k=0}^{\infty} \frac{2^{2 k}}{(k+1)^{2}\left(\begin{array}{c}
2(k+1) \\
k+1
\end{array}\right)} x^{2 k}\right] \\
& =\frac{x^{3}}{2} \sum_{n=0}^{\infty}\left[\sum_{k=0}^{n} \frac{1}{(2 k+1) 2^{2 k}}\left(\begin{array}{c}
2 k \\
k
\end{array}\right) \frac{2^{2(n-k)}}{(n-k+1)^{2}\left(\begin{array}{c}
2(n-k+1) \\
n-k+1
\end{array}\right)}\right] x^{2 n} \\
& =\sum_{n=0}^{\infty}\left[\sum_{k=0}^{n} \frac{2^{2(n-2 k)-1}}{(2 k+1)(n-k+1)^{2}} \frac{\left(\begin{array}{c}
2 k \\
k
\end{array}\right)}{\left(\begin{array}{c}
(n-k+1) \\
n-k+1
\end{array}\right)}\right] x^{2 n+3}, \\
& \frac{(\arcsin x)^{2}}{\sqrt{1-x^{2}}}=(\arcsin x)^{2} \frac{1}{\sqrt{1-x^{2}}} \\
& =\left[\frac{1}{2} \sum_{m=1}^{\infty} \frac{(2 x)^{2 m}}{m^{2}\left(\begin{array}{c}
2 m \\
m
\end{array}\right)}\right]\left[\sum_{n=0}^{\infty} \frac{1}{2^{2 n}}\left(\begin{array}{c}
2 n \\
n
\end{array}\right) x^{2 n}\right] \\
& =x^{2}\left[\sum_{k=0}^{\infty} \frac{2^{2 k+1}}{(k+1)^{2}\left(\begin{array}{c}
2(k+1) \\
k+1
\end{array}\right)} x^{2 k}\right]\left[\sum_{k=0}^{\infty} \frac{1}{2^{2 k}}\left(\begin{array}{c}
2 k \\
k
\end{array}\right) x^{2 k}\right] \\
& =\sum_{n=0}^{\infty}\left[\sum_{k=0}^{n} \frac{1}{2^{2 k}}\left(\begin{array}{c}
2 k \\
k
\end{array}\right) \frac{2^{2(n-k)+1}}{(n-k+1)^{2}\left(\begin{array}{c}
2(n-k+1) \\
n-k+1
\end{array}\right)}\right] x^{2 n+2} \\
& =\sum_{n=0}^{\infty}\left[\sum_{k=0}^{n} \frac{2^{2(n-2 k)+1}}{(n-k+1)^{2}} \frac{\left(\begin{array}{c}
2 k \\
k
\end{array}\right)}{\left(\begin{array}{c}
2(n-k+1) \\
n-k+1
\end{array}\right)}\right] x^{2 n+2} \text {, }
\end{aligned}
$$

and

$$
\begin{aligned}
\frac{(\arcsin x)^{2}}{\sqrt{1-x^{2}}} & =(\arcsin x) \frac{\arcsin x}{\sqrt{1-x^{2}}} \\
& =\left[\sum_{k=0}^{\infty} \frac{1}{2^{2 k}}\left(\begin{array}{c}
2 k \\
k
\end{array}\right) \frac{x^{2 k+1}}{2 k+1}\right]\left[\frac{1}{2 x} \sum_{m=1}^{\infty} \frac{(2 x)^{2 m}}{m\left(\begin{array}{c}
2 m \\
m
\end{array}\right)}\right] \\
& =\left[\sum_{k=0}^{\infty} \frac{1}{2^{2 k}(2 k+1)}\left(\begin{array}{c}
2 k \\
k
\end{array}\right) x^{2 k}\right]\left[\sum_{k=0}^{\infty} \frac{2^{2 k+1}}{(k+1)\left(\begin{array}{c}
2 k+1) \\
k+1
\end{array}\right)} x^{2(k+1)}\right] \\
& =\sum_{n=0}^{\infty}\left[\sum_{k=0}^{n} \frac{1}{2^{2 k}(2 k+1)}\left(\begin{array}{c}
2 k \\
k
\end{array}\right) \frac{2^{2(n-k)+1}}{(n-k+1)\left(\begin{array}{c}
2(n-k+1) \\
n-k+1
\end{array}\right)}\right] x^{2 n+2} \\
& =\sum_{n=0}^{\infty}\left[\sum_{k=0}^{n} \frac{2^{2(n-2 k)+1}}{(2 k+1)(n-k+1)} \frac{\left(\begin{array}{c}
2 k \\
k
\end{array}\right)}{\left(\begin{array}{c}
2(n-k+1) \\
n-k+1
\end{array}\right)}\right] x^{2 n+2},
\end{aligned}
$$

where we used the power series expansions (1.1), (1.2), and 2.4). Comparing the above three power series expansions with series expansions (1.3) and 3.4 and equating coefficients of $x^{2 n+2}$ respectively reveal

$$
\frac{3 ![(2 n+1) ! !]^{2}}{(2 n+3) !}\left[\sum_{k=0}^{n} \frac{1}{(2 k+1)^{2}}\right]=\sum_{k=0}^{n} \frac{2^{2(n-2 k)-1}}{(2 k+1)(n-k+1)^{2}} \frac{\left(\begin{array}{c}
2 k \\
k
\end{array}\right)}{\left(\begin{array}{c}
2(n-k+1) \\
n-k+1
\end{array}\right)},
$$




$$
\frac{2 ![(2 n+1) ! !]^{2}}{(2 n+2) !}\left[\sum_{k=0}^{n} \frac{1}{(2 k+1)^{2}}\right]=\sum_{k=0}^{n} \frac{2^{2(n-2 k)+1}}{(n-k+1)^{2}} \frac{\left(\begin{array}{c}
2 k \\
k
\end{array}\right)}{\left(\begin{array}{c}
2(n-k+1) \\
n-k+1
\end{array}\right)},
$$

and

$$
\frac{2 ![(2 n+1) ! !]^{2}}{(2 n+2) !}\left[\sum_{k=0}^{n} \frac{1}{(2 k+1)^{2}}\right]=\sum_{k=0}^{n} \frac{2^{2(n-2 k)+1}}{(2 k+1)(n-k+1)} \frac{\left(\begin{array}{c}
2 k \\
k
\end{array}\right)}{\left(\begin{array}{c}
2(n-k+1) \\
n-k+1
\end{array}\right)} .
$$

From the formula

$$
\psi^{\prime}\left(\frac{1}{2}+n\right)=\frac{\pi^{2}}{2}-4 \sum_{k=1}^{n} \frac{1}{(2 k-1)^{2}}, \quad n \in \mathbb{N}
$$

in [18, p. 914, 8.366], we derive that

$$
\sum_{k=0}^{n} \frac{1}{(2 k+1)^{2}}=\frac{1}{8}\left[\pi^{2}-2 \psi^{\prime}\left(n+\frac{3}{2}\right)\right] .
$$

Substituting the formula $(3.8)$ into $(3.5),(3.6)$, and $(3.7)$ and simplifying lead to three identities $(3.1),(3.2)$, and 3.3 respectively. The proof of Theorem 3.1 is thus complete.

\section{Remarks}

Finally, we list several remarks on our main results and related stuffs.

Remark 4.1. The identity 2.1 in Theorem 2.1 can be regarded as a couple of the identity

$$
\sum_{k=0}^{n} \frac{1}{k+1}\left(\begin{array}{c}
2 k \\
k
\end{array}\right)\left(\begin{array}{c}
2(n-k) \\
n-k
\end{array}\right)=\left(\begin{array}{c}
2 n+1 \\
n
\end{array}\right), \quad n \geq 0
$$

which is a special case of the identity [45, p. 77, (3.95)]. Moreover, the identity

$$
\sum_{\substack{k+\ell=n, k \geq 0, \ell \geq 0}} \frac{1}{k+1}\left(\begin{array}{c}
2 k \\
k
\end{array}\right)\left(\begin{array}{c}
2(\ell+1) \\
\ell+1
\end{array}\right)=2\left(\begin{array}{c}
2 n+2 \\
n
\end{array}\right), \quad n \geq 0
$$

which has been proved in [13] by three alternative and different methods, is an equivalence of the identity (4.1). This equivalence can be demonstrated as follows.

The identity 4.2 can be rearranged as

$$
\sum_{k=0}^{n} \frac{1}{k+1}\left(\begin{array}{c}
2 k \\
k
\end{array}\right)\left(\begin{array}{c}
2(n-k+1) \\
n-k+1
\end{array}\right)=2\left(\begin{array}{c}
2 n+2 \\
n
\end{array}\right)
$$

which is equivalent to

$$
\sum_{k=0}^{n+1} \frac{1}{k+1}\left(\begin{array}{c}
2 k \\
k
\end{array}\right)\left(\begin{array}{c}
2(n-k+1) \\
n-k+1
\end{array}\right)=2\left(\begin{array}{c}
2 n+2 \\
n
\end{array}\right)+\frac{1}{n+2}\left(\begin{array}{c}
2(n+1) \\
n+1
\end{array}\right)=\left(\begin{array}{c}
2 n+3 \\
n+1
\end{array}\right)
$$

where we used $\left(\begin{array}{l}0 \\ 0\end{array}\right)=1$. Replacing $n+1$ by $n$ in the last identity leads to the identity 4.1.

Remark 4.2. Closely related to central binomial coefficients $\left(\begin{array}{c}2 n \\ n\end{array}\right)$, the Catalan numbers

$$
C_{n}=\frac{1}{n+1}\left(\begin{array}{c}
2 n \\
n
\end{array}\right)
$$


in combinatorial number theory have attracted many mathematicians who have published several monographs [19, 23, 43, 47] and a number of papers such as [25, 26, 28, 29, 37, 38, 39, 40, 41, 42].

The second conclusion (b) in [3, Lemma 2] reads that

$$
\sum_{k=0}^{n} B_{k} C_{n-k}=\frac{1}{2} B_{n+1},
$$

where $B_{n}=\left(\begin{array}{c}2 n \\ n\end{array}\right)$. Rewriting the sum in 4.4 as $\sum_{k=0}^{n} B_{n-k} C_{k}$ and substituting $\left(\begin{array}{c}2 n-2 k \\ n-k\end{array}\right)$ and $\frac{1}{k+1}\left(\begin{array}{c}2 k \\ k\end{array}\right)$ for $B_{n-k}$ and $C_{k}$ result in

$$
\sum_{k=0}^{n} \frac{1}{k+1}\left(\begin{array}{c}
2 k \\
k
\end{array}\right)\left(\begin{array}{c}
2(n-k) \\
n-k
\end{array}\right) C_{k}=\frac{1}{2}\left(\begin{array}{c}
2(n+1) \\
n+1
\end{array}\right)=\left(\begin{array}{c}
2 n+1 \\
n
\end{array}\right)
$$

which is the same as the identity 4.1).

By the way, the combinatorial proof of the identity (4.4) in [3, Lemma 2] is longer than the combinatorial proof of the identity (4.2) in [13, while its equivalent identities 4.1) and 4.2 were proved analytically in [13] and [45, p. 77, (3.95)].

Remark 4.3. By the formula 4.3, we can rewritten the identity 4.1 and those in Theorem 2.1 and Theorem 3.1 as

$$
\begin{aligned}
\sum_{k=0}^{n}(n-k+1) C_{k} C_{n-k} & =\left(\begin{array}{c}
2 n+1 \\
n
\end{array}\right), \\
\sum_{k=0}^{n} \frac{(k+1)(n-k+1)}{2 k+1} C_{k} C_{n-k} & =\frac{2^{4 n}}{(2 n+1)(n+1) C_{n}}, \\
\sum_{k=0}^{n} \frac{n-k+2}{2^{4 k}(k+1)(2 k+1)(n-k+1)^{2}} \frac{C_{k}}{C_{n-k+1}} & =\frac{3[(2 n+1) ! !]^{2}}{2^{2 n}(2 n+3) !} \sum_{k=0}^{n} \frac{1}{(2 k+1)^{2}}, \\
\sum_{k=0}^{n} \frac{n-k+2}{2^{4 k}(k+1)(n-k+1)^{2}} \frac{C_{k}}{C_{n-k+1}} & =\frac{[(2 n+1) ! !]^{2}}{2^{2 n}(2 n+2) !} \sum_{k=0}^{n} \frac{1}{(2 k+1)^{2}}
\end{aligned}
$$

and

$$
\sum_{k=0}^{n} \frac{n-k+2}{2^{4 k}(k+1)(2 k+1)(n-k+1)} \frac{C_{k}}{C_{n-k+1}}=\frac{[(2 n+1) ! !]^{2}}{2^{2 n}(2 n+2) !} \sum_{k=0}^{n} \frac{1}{(2 k+1)^{2}}
$$

respectively. For more information on series involving the Catalan numbers $C_{n}$, please refer to the paper [34] and closely related references therein.

Remark 4.4. In [21] and its previous arXiv preprints, among other things, a nice series expansion and its applications of the function $\left(\frac{\arcsin t}{t}\right)^{m}$, whose value at $t=0$ is defined to be 1 , were established and carried out.

Remark 4.5. This paper is a revised version of the arXiv preprints [30, 31].

\section{Acknowledgements}

The authors thank

1. Nguyen Xuan Tho (Hanoi University of Science and Technology, Vietnam) for supplying the reference [13] on 9 November 2020;

2. Mikhail Yu. Kalmykov (Bogoliubov Laboratory of Theoretical Physics, Joint Institute for Nuclear Research, Russia; kalmykov.mikhail@googlemail.com) for providing the papers [14, 15, 22] on 9 January 2021;

3. anonymous referees for pointing out or reminding of the papers [3, 45, 49]. 


\section{Conflict of interest}

The authors declare that they have no conflict of interest.

\section{References}

[1] M. Abramowitz and I. A. Stegun (Eds), Handbook of Mathematical Functions with Formulas, Graphs, and Mathematical Tables, National Bureau of Standards, Applied Mathematics Series 55, 10th printing, Dover Publications, New York and Washington, 1972.

[2] E. P. Adams and R. L. Hippisley, Smithsonian Mathematical Formulae and Tables of Elliptic Functions, Smithsonian Institute, Washington, D.C., 1922.

[3] H. Alzer and G. V. Nagy, Some identities involving central binomial coefficients and Catalan numbers, Integers 20 (2020), Paper No. A59, 17 pages.

[4] B. C. Berndt, Ramanujan's Notebooks, Part I, With a foreword by S. Chandrasekhar, Springer-Verlag, New York, 1985; available online at https://doi.org/10.1007/978-1-4612-1088-7.

[5] J. M. Borwein, D. H. Bailey, and R. Girgensohn, Experimentation in Mathematics: Computational Paths to Discovery, A K Peters, Ltd., Natick, MA, 2004.

[6] J. M. Borwein and P. B. Borwein, Pi and the AGM: A Study in Analytic Number Theory and Computational Complexity, Canadian Mathematical Society Series of Monographs and Advanced Texts, A Wiley-Interscience Publication, John Wiley \& Sons, Inc., New York, 1987.

[7] K. N. Boyadzhiev, Series with central binomial coefficients, Catalan numbers, and harmonic numbers. J. Integer Seq. 15 (2012), no. 1, Article 12.1.7, 11 pp.

[8] D. M. Bradley, A class of series acceleration formulae for Catalan's constant, Ramanujan J. 3 (1999), no. 2, 159-173; available online at https://doi.org/10.1023/A:1006945407723

[9] T. J. I. Bromwich, An Introduction to the Theory of Infinite Series, Macmillan and Co., Limited, London, 1908.

[10] J. M. Campbell, New series involving harmonic numbers and squared central binomial coefficients, Rocky Mountain J. Math. 49 (2019), no. 8, 2513-2544; available online at https://doi.org/10.1216/RMJ-2019-49-8-2513

[11] H. Chen, Interesting series associated with central binomial coefficients, Catalan numbers and harmonic numbers, J. Integer Seq. 19 (2016), no. 1, Article 16.1.5, 11 pp.

[12] C.-P. Chen, Sharp Wilker- and Huygens-type inequalities for inverse trigonometric and inverse hyperbolic functions, Integral Transforms Spec. Funct. 23 (2012), no. 12, 865-873; available online at https://doi.org/10.1080/10652469.2011.644851

[13] P. P. Dályay, Y. J. Ionin, O. P. Lossers, and J. H. Smith, A product of Catalan numbers, Amer. Math. Monthly 125 (2018), no. 1, 86-87; available online at https://doi.org/10.1080/00029890.2018.1397465.

[14] A. I. Davydychev and M. Yu. Kalmykov, Massive Feynman diagrams and inverse binomial sums, Nuclear Phys. B 699 (2004), no. 1-2, 3-64; available online at https://doi.org/10.1016/j.nuclphysb.2004.08.020

[15] A. I. Davydychev and M. Yu. Kalmykov, New results for the $\varepsilon$-expansion of certain one-, two- and three-loop Feynman diagrams, Nuclear Phys. B 605 (2001), no. 1-3, 266-318; available online at https://doi.org/10.1016/S0550-3213(01) 00095-5

[16] J. Edwards, Differential Calculus, 2nd ed., Macmillan, London, 1982.

[17] M. Garcia-Armas and B. A. Seturaman, A note on the Hankel transform of the central binomial coefficients, J. Integer Seq. 11(2008), Article 08.5.8, 9 pages.

[18] I. S. Gradshteyn and I. M. Ryzhik, Table of Integrals, Series, and Products, Translated from the Russian, Translation edited and with a preface by Daniel Zwillinger and Victor Moll, Eighth edition, Revised from the seventh edition, Elsevier/Academic Press, Amsterdam, 2015; available online at https://doi.org/10.1016/B978-0-12-384933-5.00013-8

[19] R. P. Grimaldi, Fibonacci and Catalan Numbers, John Wiley \& Sons, Inc., Hoboken, NJ, 2012; available online at https: //doi.org/10.1002/9781118159743

[20] C.-Y. Gu and V. J. W. Guo, Proof of two conjectures on supercongruences involving central binomial coefficients, Bull. Aust. Math. Soc. 102 (2020), no. 3, 360-364; available online at https://doi.org/10.1017/s0004972720000118

[21] B.-N. Guo, D. Lim, and F. Qi, Maclaurin series expansions for powers of inverse (hyperbolic) sine, for powers of inverse (hyperbolic) tangent, and for incomplete gamma functions, with applications to second kind Bell polynomials and generalized logsine function, arXiv preprint (2021), available online at https://arxiv.org/abs/2101.10686v5.

[22] M. Yu. Kalmykov and A. Sheplyakov, lsjk-a $C++$ library for arbitrary-precision numeric evaluation of the generalized log-sine functions, Computer Phys. Commun. 172 (2005), no. 1, 45-59; available online at https://doi.org/10.1016/j. cpc.2005.04.013

[23] T. Koshy, Catalan Numbers with Applications, Oxford University Press, Oxford, 2009.

[24] D. H. Lehmer, Interesting series involving the central binomial coefficient, Amer. Math. Monthly 92 (1985), no. 7, 449-457; available online at http://dx.doi.org/10.2307/2322496

[25] W.-H. Li, F. Qi, O. Kouba, and I. Kaddoura, A further generalization of the Catalan numbers and its explicit formula and integral representation, OSF Preprints (2020), available online at https://doi.org/10.31219/osf.io/zf9xu

[26] M. Mahmoud and F. Qi, Three identities of the Catalan-Qi numbers, Mathematics 4 (2016), no. 2, Article 35, 7 pages; available online at https://doi.org/10.3390/math4020035

[27] J. Mikić, On certain sums divisible by the central binomial coefficient, J. Integer Seq. 23 (2020), no. 1, Art. 20.1.6, 22 pages. 
[28] F. Qi, Some properties of the Catalan numbers, Ars Combin. (2022), in press; available online at https://www. researchgate.net/publication/328891537.

[29] F. Qi and P. Cerone, Some properties of the Fuss-Catalan numbers, Mathematics 6 (2018), no. 12, Article 277, 12 pages; available online at https://doi.org/10.3390/math6120277.

[30] F. Qi, C.-P. Chen, and D. Lim, Five identities involving the product or ratio of two central binomial coefficients, arXiv preprint (2021), available online at https://arxiv.org/abs/2101.02027.

[31] F. Qi, C.-P. Chen, and D. Lim, Several combinatorial identities derived from series expansions of powers of arcsine, arXiv preprint (2021), available online at https://arxiv.org/abs/2101.02027v2.

[32] F. Qi and B.-N. Guo, From inequalities involving exponential functions and sums to logarithmically complete monotonicity of ratios of gamma functions, J. Math. Anal. Appl. 493 (2021), no. 1, Article 124478, 19 pages; available online at https://doi.org/10.1016/j.jmaa.2020.124478

[33] F. Qi and B.-N. Guo, Integral representations of the Catalan numbers and their applications, Mathematics 5 (2017), no. 3, Article 40, 31 pages; available online at https://doi.org/10.3390/math5030040

[34] F. Qi and B.-N. Guo, Sums of infinite power series whose coefficients involve products of the Catalan-Qi numbers, Montes Taurus J. Pure Appl. Math. 1 (2019), no. 2, Article ID MTJPAM-D-19-00007, 1-12.

[35] F. Qi, W.-H. Li, J. Cao, D.-W. Niu, and J.-L. Zhao, An analytic generalization of the Catalan numbers and its integral representation, arXiv preprint (2020), available online at https://arxiv.org/abs/2005.13515v1

[36] F. Qi, W.-H. Li, S.-B. Yu, X.-Y. Du, and B.-N. Guo, A ratio of many gamma functions and its properties with applications, Rev. R. Acad. Cienc. Exactas Fís. Nat. Ser. A Math. RACSAM. 115 (2021), no. 2, Paper No. 39, 14 pages; available online at https://doi.org/10.1007/s13398-020-00988-z.

[37] F. Qi, M. Mahmoud, X.-T. Shi, and F.-F. Liu, Some properties of the Catalan-Qi function related to the Catalan numbers, SpringerPlus 5 (2016), Paper No. 1126, 20 pages; available online at https://doi.org/10.1186/s40064-016-2793-1.

[38] F. Qi, X.-T. Shi, and F.-F. Liu, An integral representation, complete monotonicity, and inequalities of the Catalan numbers, Filomat 32 (2018), no. 2, 575-587; available online at https://doi.org/10.2298/FIL1802575Q.

[39] F. Qi, X.-T. Shi, F.-F. Liu, and D. V. Kruchinin, Several formulas for special values of the Bell polynomials of the second kind and applications, J. Appl. Anal. Comput. 7 (2017), no. 3, 857-871; available online at https://doi .org/10.11948/2017054

[40] F. Qi, X.-T. Shi, M. Mahmoud, and F.-F. Liu, The Catalan numbers: a generalization, an exponential representation, and some properties, J. Comput. Anal. Appl. 23 (2017), no. 5, 937-944.

[41] F. Qi and Y.-H. Yao, Simplifying coefficients in differential equations for generating function of Catalan numbers, J. Taibah Univ. Sci. 13 (2019), no. 1, 947-950; available online at https://doi.org/10.1080/16583655.2019.1663782

[42] F. Qi, Q. Zou, and B.-N. Guo, The inverse of a triangular matrix and several identities of the Catalan numbers, Appl. Anal. Discrete Math. 13 (2019), no. 2, 518-541; available online at https://doi.org/10.2298/AADM190118018Q.

[43] S. Roman, An Introduction to Catalan Numbers, with a foreword by Richard Stanley, Compact Textbook in Mathematics, Birkhäuser/Springer, Cham, 2015; available online at https://doi.org/10.1007/978-3-319-22144-1

[44] M. Z. Spivey, The Art of Proving Binomial Identities, Discrete Mathematics and its Applications (Boca Raton), CRC Press, Boca Raton, FL, 2019; available online at https://doi.org/10.1201/9781351215824

[45] R. Sprugnoli, Riordan Array Proofs of Identities in Gould's Book, University of Florence, Italy, 2006.

[46] R. Sprugnoli, Sums of reciprocals of the central binomial coefficients, Integers 6 (2006), A27, 18 pp.

[47] R. P. Stanley, Catalan Numbers, Cambridge University Press, New York, 2015; available online at https://doi.org/10. 1017/CB09781139871495

[48] H. S. Wilf, generatingfunctionology, Third edition. A K Peters, Ltd., Wellesley, MA, 2006.

[49] R. Witula, E. Hetmaniok, D. Słota, and N. Gawrońska, Convolution identities for central binomial numbers, Int. J. Pure App. Math. 85 (2013), no. 1, 171-178; available online at https://doi.org/10.12732/ijpam.v85i1.14.

[50] B. Zhang and C.-P. Chen, Sharp Wilker and Huygens type inequalities for trigonometric and inverse trigonometric functions, J. Math. Inequal. 14 (2020), no. 3, 673-684; available online at https://doi.org/10.7153/jmi-2020-14-43 